\title{
La préparation colique avant chirurgie colorectale : et si on avait eu tout faux (et tous faux...)?
}

\author{
Y. Panis \\ (C) Lavoisier SAS 2017
}

L'infection du site opératoire (ISO) est une cause majeure de morbidité postopératoire après chirurgie colorectale, avec des taux variant de 8 à $40 \%$ [1], le site opératoire pouvant être facilement contaminé par des agents bactériologiques pathogènes issus du tractus digestif. L'apparition d'une ISO a été identifiée comme un facteur de risque indépendant de mortalité postopératoire et est associée à une augmentation significative de la durée d'hospitalisation, du taux de réhospitalisation, et du taux de réintervention, aboutissant à une multiplication des coûts de soins par un facteur de 2 à 3 . La prévention de l'apparition des ISO est ainsi un point clé de la sécurité du patient en chirurgie colorectale.

La prévention de l'apparition des ISO en chirurgie colorectale est basée sur le contrôle optimal de ses facteurs de risque modifiables. Ces facteurs de risque peuvent être liés au patient et liés à l'intervention chirurgicale. Les facteurs de risqué liés au patient incluent les comorbidités associées, l'état nutritionnel, l'obésité, le tabagisme, le diabète, l'anémie préopératoire et l'immunosuppression [1]. Les facteurs liés à l'intervention incluent l'immunonutrition préopératoire, l'antisepsie cutanée, le contrôle peropératoire de la température corporelle, l'asepsie chirurgicale, l'hémostase, la voie d'abord chirurgicale et la durée de l'intervention [2].

Dans ce contexte, la préparation colique préopératoire (PCP) a été proposée avant chirurgie colorectale afin de réduire les selles dans le tube digestif et ainsi limiter le risque de contamination du champ opératoire par des agents bactériologiques pathogènes issus du tractus digestif. Le bénéfice de la PCP est cependant variable en fonction du type d'intervention réalisée. Ainsi, plusieurs essais randomisés et métaanalyses, dont une réalisée par la fondation de recherche Cochrane, ont suggéré l'absence de bénéfice de la PCP sur le taux de morbidité postopératoire avant chirurgie élective du cancer du côlon [3]. Une méta-analyse d'essais randomisés, qui comparait la PCP à l'absence de PCP avant chirurgie

\footnotetext{
Y. Panis $(\bowtie)$
}

Service de chirurgie colorectale, pôle des maladies de l'appareil digestif, hôpital Beaujon - Assistance Publique des Hôpitaux de Paris (APHP), université Paris VII (Denis Diderot), 100 boulevard du Général Leclerc, F-92118 Clichy cedex e-mail : yves.panis@bjn.aphp.fr élective du cancer du côlon, à même suggéré que la réalisation d'une PCP pouvait en fait être à l'origine d'une augmentation du taux d'ISO postopératoire (odds ratio : 1,40 [1,05$1,87] ; p=0,02$ ) [4]. Ces résultats ont amené les recommandations françaises à proposer de ne pas réaliser de PCP avant la chirurgie élective du cancer du côlon [5].

À l'inverse, la PCP pourrait permettre de réduire le risque d'ISO après chirurgie élective du cancer du rectum. Nous avons en effet conduit un essai randomisé multicentrique français qui comparait la PCP à l'absence de PCP chez les patients opérés électivement d'un cancer du rectum. Cet essai a démontré que la PCP réduisait significativement le taux d'ISO après chirurgie du rectum de $39 \%$ (incluant $38 \%$ d'ISO profondes et $1 \%$ d'ISO superficielles) dans le groupe sans PCP à $20 \%$ (incluant $17 \%$ d'ISO profondes et $3 \%$ d'ISO superficielles) dans le groupe avec PCP [6]. Les résultats de cet essai ont amené les recommandations françaises à proposer la réalisation systématique d'une PCP avant chirurgie élective du cancer du rectum [7].

Cependant, plusieurs études récentes ont suggéré que l'adjonction d'antibiotiques oraux à la PCP pouvait améliorer la décontamination digestive et ainsi diminuer le taux d'ISO après chirurgie colorectale. En effet, une méta-analyse d'essais randomisés récente a suggéré que les patients recevant une combinaison de PCP et d'antibiotiques oraux avant chirurgie colorectale étaient exposés à un risque diminué d'ISO par rapport aux patients ne recevant qu'une PCP seule (risqué relatif : $0,45[0,34-0,60] ; \mathrm{p}<0,001)$ [8]. Ce résultat a été confirmé dans un essai randomisé récent qui comparait PCP avec antibiotiques oraux à PCP seule dans une population hétérogène de patients opérés d'une résection laparoscopique du côlon ou du rectum [9]. Cette étude rapportait une réduction de $50 \%$ du taux d'ISO dans le groupe PCP et antibiotiques oraux par rapport au groupe PCP seule (respectivement $7,26 \%$ contre $12,8 \%$ ) [9]. Enfin, trois récentes études de registre, incluant chacune plus de 4000 patients opérés d'une résection laparoscopique du côlon ou du rectum, ont rapporté les résultats de la stratégie d'adjonction d'antibiotiques oraux à la PCP [10-12]. Leurs résultats étaient similaires et suggéraient que le taux d'ISO postopératoire le plus faible était observé chez les patients ayant reçu une combinaison de 
PCP et d'antibiotiques oraux $(6,2 \%)$ par rapport aux patients n'ayant reçu qu'une PCP seule $(12,1 \%)$ [10].

À ce jour, aucun essai randomisé n'a cependant comparé les résultats d'une $\mathrm{PCP}$ avec antibiotiques oraux à ceux d'une PCP seule dans une population homogène de patients opérés du rectum par voie laparoscopique, qui est actuellement la voie d'abord de référence. La grande majorité des essais disponibles n'incluaient en effet que des patients opérés par voie ouverte, à l'origine d'un taux de complications postopératoires augmenté [8]. Un seul essai randomisé récent n'incluait que des patients opérés par laparoscopie mais avec une population hétérogène de patient opérés du côlon ou du rectum [9], alors qu'il est démontré que l'impact de la réalisation d'une PCP est différent en fonction du type de chirurgie réalisé.

C'est la raison pour laquelle nous avons proposé dans le cadre du programme PHRC de recherche clinique le premier essai randomisé en double aveugle comparant les résultats de la PCP avec antibiotiques oraux à ceux de la PCP seule chez les patients opérés du rectum par voie laparoscopique avec deux bras d'étude : un groupe avec PCP seule et un groupe avec PCP et antibiotiques oraux. Les antibiotiques oraux incluront la gentamicine orale ( $80 \mathrm{mg} \mathrm{x} 4$ par jour) et l'ornidazole oral (1 g par jour) pendant les deux jours précédant l'intervention chirurgicale, dans le même temps que la PCP. Les patients inclus dans le groupe sans antibiotique oral recevront des placebos oraux pendant les deux jours précédant l'intervention chirurgicale. De même, nous avons proposé une étude randomisée similaire pour la chirurgie du cancer du côlon mais à quatre bras cette fois ci : aucune préparation, PCP seule, antibiotiques oraux seuls, et PCP + antibiotiques oraux.

L'objectif principal de ces deux études sera de comparer le taux d'ISO profonde et superficielle postopératoire entre les différents groupes (en espérant une réduction du taux d'ISO de 15 à $7,5 \%$ dans la chirurgie du cancer du côlon). La réduction du taux d'ISO postopératoire pourrait permettre : (a) à court terme : une réduction du taux de complications postopératoires et de la nécessité de ré-intervention, de prolongement de l'hospitalisation, et de ré-hospitalisation. De plus, à un niveau de santé publique, la diminution du taux d'ISO pourrait avoir un impact significatif sur le coût de la chirurgie du cancer colorectal ; (b) à long terme : il est maintenant bien démontré que l'apparition d'une ISO a un impact important sur les patients opérés d'un cancer du rectum en augmentant le risque de stomie définitive et en péjorant les résultats fonctionnels des patients sans stomie [13]. Enfin, une méta-analyse récente a suggéré que l'apparition d'une ISO pouvait être à l'origine de résultats oncologiques péjorés avec une augmentation du risque de récidives locales [14].

En conclusion, la « fin de l'histoire » de la préparation colique n'est apparemment pas arrivée. Dans le cadre notamment des protocoles de réhabilitation précoce, l'absence de préparation colique était considérée comme un signe de simplification de la prise en charge au bénéfice du patient. Les études qui vont débuter vont peut être complétement modifier cette vision des choses en revenant à une préparation colique pour tout les patients opérés du côlon et du rectum !

Liens d'intérêts : L'auteur déclare ne pas avoir de lien d'intérêt.

\section{Références}

1. Hubner M, Diana M, Zanetti G, et al (2011) Surgical site infections in colon surgery: the patient, the procedure, the hospital, and the surgeon. Arch Surg 146:1240-5

2. Tang R, Chen HH, Wang YL, et al (2001) Risk factors for surgical site infection after elective resection of the colon and rectum: a single-center prospective study of 2,809 consecutive patients. Ann Surg 234:181-9

3. Guenaga KF, Matos D, Wille-Jorgensen P (2011) Mechanical bowel preparation for elective colorectal surgery. Cochrane Database Syst Rev 2011:CD001544

4. Slim K, Vicaut E, Launay-Savary MV, et al (2009) Updated systematic review and meta-analysis of randomized clinical trials on the role of mechanical bowel preparation before colorectal surgery. Ann Surg 249:203-9

5. Mariette C, Alves A, Benoist S, et al (2005) [Perioperative care in digestive surgery. Guidelines for the French society of digestive surgery (SFCD)]. Ann Chir 130: 08-24

6. Bretagnol F, Panis Y, Rullier E, et al (2010) Rectal cancer surgery with or without bowel preparation: The French GRECCAR III multicenter single-blinded randomized trial. Ann Surg 252:863-8

7. Bridoux V, de Chaisemartin C, Beyer L, et al (2016) Recommandations pour la pratique clinique: cancer du rectum. Quels sont les critères de qualité de l'exérèse chirurgicale ? Côlon Rectum 10:12-27

8. Chen M, Song X, Chen LZ, et al (2016) Comparing Mechanical Bowel Preparation With Both Oral and Systemic Antibiotics Versus Mechanical Bowel Preparation and Systemic Antibiotics Alone for the Prevention of Surgical Site Infection After Elective Colorectal Surgery: A Meta-Analysis of Randomized Controlled Clinical Trials. Dis Colon Rectum 59:70-8

9. Hata H, Yamaguchi T, Hasegawa S, et al (2016) Oral and Parenteral Versus Parenteral Antibiotic Prophylaxis in Elective Laparoscopic Colorectal Surgery (JMTO PREV 07-01): A Phase 3, Multicenter, Open-label, Randomized Trial. Ann Surg 263:1085-91

10. Kiran RP, Murray AC, Chiuzan C, et al (2015) Combined preoperative mechanical bowel preparation with oral antibiotics significantly reduces surgical site infection, anastomotic leak, and ileus after colorectal surgery. Ann Surg 262:416-25

11. Scarborough JE, Mantyh CR, Sun Z, Migaly J (2015) Combined Mechanical and Oral Antibiotic Bowel Preparation Reduces Incisional Surgical Site Infection and Anastomotic Leak Rates After Elective Colorectal Resection: An Analysis of ColectomyTargeted ACS NSQIP. Ann Surg 262:331-7

12. Morris MS, Graham LA, Chu DI, et al (2015) Oral Antibiotic Bowel Preparation Significantly Reduces Surgical Site Infection Rates and Readmission Rates in Elective Colorectal Surgery. Ann Surg 261:1034-40

13. den Dulk M, Smit M, Peeters KC, et al (2007) A multivariate analysis of limiting factors for stoma reversal in patients with rectal cancer entered into the total mesorectal excision (TME) trial: a retrospective study. Lancet Oncol 8:297-303

14. Mirnezami A, Mirnezami R, Chandrakumaran K, et al (2011) Increased local recurrence and reduced survival from colorectal cancer following anastomotic leak: systematic review and metaanalysis. Ann Surg 253:890-9 